\title{
Diversity in the dynamical behaviour of a compartmentalized programmable biochemical oscillator
}

\author{
Maximilian Weitz ${ }^{1}$, Jongmin Kim $^{2}$, Korbinian Kapsner, Erik Winfree ${ }^{2,3,4}$, Elisa Franco ${ }^{5 \star}$ \\ and Friedrich C. Simmel ${ }^{1 \star}$
}

\begin{abstract}
In vitro compartmentalization of biochemical reaction networks is a crucial step towards engineering artificial cell-scale devices and systems. At this scale the dynamics of molecular systems becomes stochastic, which introduces several engineering challenges and opportunities. Here we study a programmable transcriptional oscillator system that is compartmentalized into microemulsion droplets with volumes between $33 \mathrm{fl}$ and $16 \mathrm{pl}$. Simultaneous measurement of large populations of droplets reveals major variations in the amplitude, frequency and damping of the oscillations. Variability increases for smaller droplets and depends on the operating point of the oscillator. Rather than reflecting the stochastic kinetics of the chemical reaction network itself, the variability can be attributed to the statistical variation of reactant concentrations created during their partitioning into droplets. We anticipate that robustness to partitioning variability will be a critical challenge for engineering cell-scale systems, and that highly parallel time-series acquisition from microemulsion droplets will become a key tool for characterization of stochastic circuit function.
\end{abstract}

B ological networks can perform highly sophisticated tasks such as signal processing, computation and the orchestration of molecular processes in time and space. In the past decade a variety of increasingly complex artificial, programmable molecular circuits have been demonstrated in vitro. On a fundamental level, such systems can be used to prototype and analyse subsystems of more-complicated naturally occurring circuits, and thus serve as a training ground for understanding biological complexity. Beyond biology, in vitro molecular systems offer considerable design flexibility using a limited number of well-characterized components ${ }^{1-3}$. Thus, they constitute an ideal platform for developing nanosystems that operate in a cell-free environment and exploit attractive features of cellular machinery, such as the ability to replicate, self-assemble and compute at the nanoscale.

An important step towards engineering compact biomimetic systems is the encapsulation of biochemical circuitry within celllike microcompartments-the creation of programmable 'artificial cells' or 'protocells'. However, compartmentalization can profoundly influence biochemical reaction kinetics ${ }^{4,5}$. In particular, typical concentrations of molecules in biomolecular circuits are in the nanomolar range, which for cell-sized reaction containers means that some molecular species are present only at very small copy numbers. A prominent consequence of this is the appearance of biochemical 'noise', often attributed to the inherent stochasticity of chemical reactions ${ }^{6}$. Understanding the sources and propagation of noise and variability in artificial biochemical systems encapsulated in microcompartments is necessary to provide a foundation for engineering molecular systems that are robust to stochasticity, or that exploit the randomness to advantage. Furthermore, because biological cells face many of the same challenges and opportunities, our understanding of biological systems can be informed by, and can provide insight into, engineering principles for molecular systems at this scale.

Cell-free gene expression in lipid bilayer vesicles has been studied in the past, but functional encapsulation of complex mixtures of biochemicals, such as the transcription/translation machinery, remains challenging ${ }^{7,8}$. A technologically more-developed approach employs water-in-oil emulsion droplets as reaction containers ${ }^{5,9,10}$, with applications that include single-molecule enzymology ${ }^{11}$, emulsion polymerase chain reaction (PCR) ${ }^{12}$ and in vitro evolution experiments for the selection of ribozymes or functional proteins ${ }^{13,14}$. However, thus far the influence of micron-scale encapsulation on in vitro biochemical networks with more-complex, far from equilibrium, dynamical behaviour has not been investigated systematically.

Previously, compartmentalization of an inorganic chemical dynamical process was demonstrated with the BelousovZhabotinsky reaction, where the emergence of oscillations and spatial patterns was studied in microemulsion droplets ${ }^{15}$. As inorganic chemical oscillators operate at much higher concentrations than typical biochemical systems, fluctuations and 'small-number effects' were, with few exceptions ${ }^{16,17}$, typically not observed. Recently, a range of synthetic biochemical oscillators have been demonstrated successfully both in vivo ${ }^{18-22}$ and in vitro ${ }^{3,23-25}$. Owing to their important role in the orchestration of biological processes $^{26,27}$, there is considerable interest in the robustness of naturally occurring biochemical clocks with respect to molecular noise $\mathrm{e}^{27-29}$ or temperature fluctuations ${ }^{30}$. So far, synthetic in vitro biochemical oscillators have been studied only in bulk reactions $s^{3,24,25,31}$, or encapsulated into emulsion droplets too large to result in considerable dynamic variability ${ }^{32}$.

Here, we demonstrate and characterize compartmentalization of a synthetic biochemical clock (a fluorescent in vitro transcriptional

\footnotetext{
'Systems Biophysics and Bionanotechnology, Physik Department and WSI/ZNN, Technische Universität München, 85748 Garching, Germany, ${ }^{2}$ Bioengineering, California Institute of Technology, Pasadena, California 91125, USA, ${ }^{3}$ Computation and Neural Systems, California Institute of Technology, Pasadena, California 91125, USA, ${ }^{4}$ Computer Science, California Institute of Technology, Pasadena, California 91125, USA, ${ }^{5}$ Mechanical Engineering, University of California at Riverside, Riverside, California 92521, USA. *e-mail: efranco@engr.ucr.edu; simmel@tum.de
} 
oscillator) into small, cell-sized droplet microreactors. The oscillator reaction system involves seven DNA strands, two enzymes, two transcribed RNA species and several intermediate species. Using a simple vortexing technique, we generated emulsions of water-inoil droplets that contained the oscillator, with volumes ranging from $\gtrsim 16 \mathrm{pl}$ down to $\lesssim 33 \mathrm{fl}$. Thousands of individual oscillating droplets were followed simultaneously by optical microscopy, and features such as the period and amplitude of each droplet's fluorescence trace were measured using automated analysis procedures.

As anticipated for small-number effects, the diversity of behaviours increased in smaller droplets. Surprisingly, however, we found that the diversity could not be explained by the inherent stochasticity of chemical reactions that involve small numbers of molecules, which is often used as an explanation for variability within living cells $\mathrm{s}^{33,34}$. Instead, our experimental data were more consistent with models in which stochastic partitioning of key low-concentration species (enzymes in particular) introduced diversity in the dynamical behaviour by providing variability in the initial conditions for the oscillator. Thus, the variability observed in our population of oscillators bears more similarities to the 'cell-division noise' caused by the unequal distribution of the molecules of dividing cells between their daughter cells ${ }^{35,36}$.

\section{Results and discussion}

A synthetic transcriptional oscillator. Schematic representations of the in vitro transcriptional oscillator used in the present study are shown in Fig. 1a,b. Its operation principle and quantitative description are discussed thoroughly by Kim and Winfree ${ }^{24}$ and Franco et al. ${ }^{25}$. Briefly, it is based on two transcriptional switches, SW21 and SW12 (also termed 'genelets'), that mutually regulate their activity through RNA transcripts rA1 (activator) and rI2 (inhibitor). The genelets are composed of double-stranded DNA templates (T21A1 and T12A2) that contain the promoter sequence of RNA polymerase (RNAP) from bacteriophage T7. The non-coding strand of the genelets is nicked in the promoter region, and removal of the activating DNA strands A1 or A2 by toehold-mediated strand displacement results in strongly reduced transcriptional activity. RNA species rI2, which is transcribed from T21A1, can inhibit transcription from genelet SW12 by displacing A2 from T12A2. By contrast, RNA species rA1, transcribed from T12A2, can activate genelet SW21 by displacing dI1 strands from A1dI1 duplexes; A1 strands are thus released, and can bind to T21. As a result, the two genelets constitute an overall negative feedback loop, which exhibits oscillatory behaviour for appropriate parameter settings. To prevent unlimited growth of RNA concentrations, RNase $\mathrm{H}$ is added to degrade, both selectively and processively, RNA that occurs in RNA/DNA hybrid duplexes ${ }^{37}$.

Experimentally, the state of the genelets is read out by fluorescence collected from the dye-labelled T21 strands. A typical fluorescence trace recorded from the transcriptional oscillator system is shown in Fig. 1c. As shown in the figure, experimental data are well reproduced by a theoretical model (the extended model from Kim and Winfree ${ }^{24}$ ) that captures the most-important chemical reactions (Supplementary Section Modelling). The model contains 17 ordinary differential equations with 24 species and 24 rate parameters. The model parameters were chosen within a realistic range to obtain a least-squares fit to data collected from three distinct operating points of the oscillator, which we named 'sustained' (Fig. 1c), 'damped' and 'strongly damped'.

The enzyme concentrations for the 'sustained' tuning of the oscillator system are $[$ RNase $\mathrm{H}] \approx 5 \mathrm{nM}$ and $[\mathrm{RNAP}] \approx 200 \mathrm{nM}$, and from $80 \mathrm{nM}$ to $500 \mathrm{nM}$ for the oligonucleotide components (Supplementary Section Methods). In droplets with subpicolitre volumes, some of the species are present at only relatively low copy numbers. For instance, in a droplet with radius $(r)$ of $2 \mu \mathrm{m}$
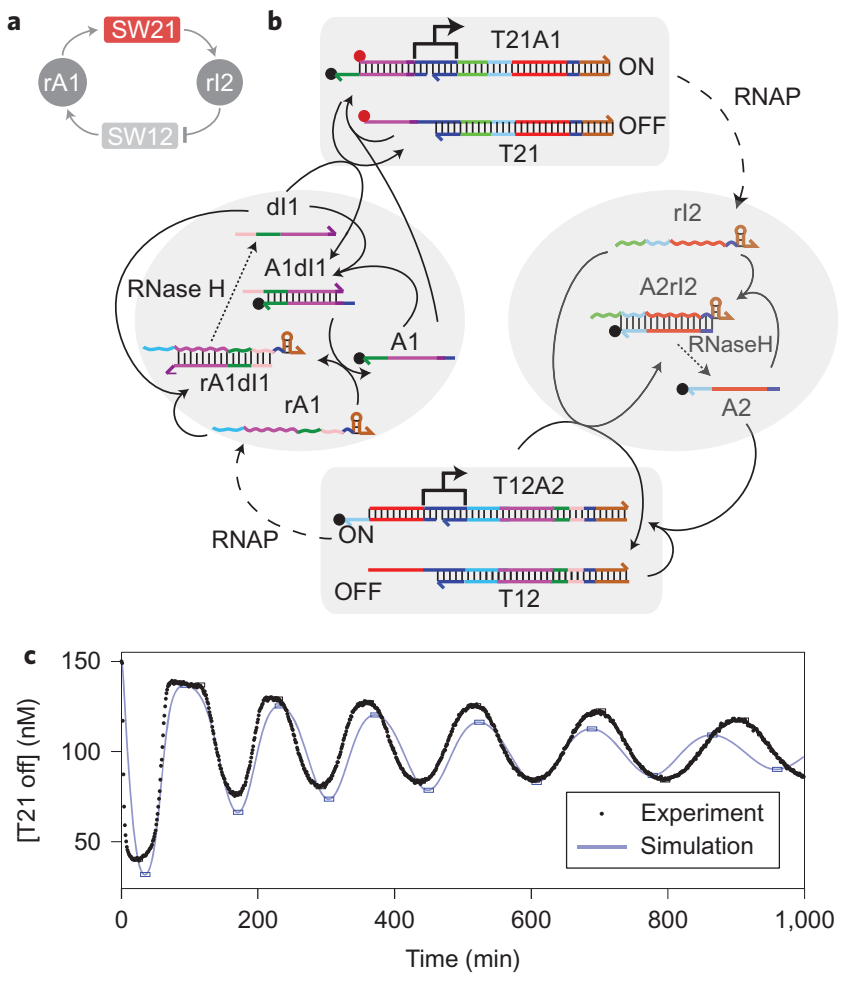

Figure 1 | An in vitro transcriptional oscillator. a, Schematic representation of the two-switch negative-feedback oscillator circuit. Arrowheads indicate activation of a downstream component, and the blunt end indicates inhibition. b, Molecular representation of involved reactions. DNA strands are represented by coloured lines. Complementary sub-sequences are coloured identically. RNA signals are represented by wavy lines. Black dashed lines connect transcription substrate and product, and dotted lines connect degradation substrates and remaining products. Switches SW12 (SW21) from a consist of genelets T12 (T21) that can be switched to a transcriptional active state by hybridization of an activator strand A2 (A1), which completes the promoter sequence for T7 RNAP (boxes with arrows indicate the direction of transcription). Inhibitor strands dl1 (rl2), which are complementary to activators, switch transcription off by toehold-mediated strand displacement. Excesses of activator A2 and inhibitor d11 act as thresholds by sequestering free RNA signals up to a certain level, and thus cause a delay in the negative feedback loop that leads to oscillatory behaviour. Genelet T21 is labelled with a fluorophore (red circle) and A1 is labelled with a quencher (black circle), which results in high fluorescence for low transcription activity and vice versa. DNA sequences are given in the Supplementary Methods. c, The fluorescent time trace of switch T21 (black) and corresponding fit of the extended model (blue) of the oscillator circuit exhibit sustained oscillations. The eventual decay of oscillator amplitude and period is attributed to the build-up of incomplete RNA degradation products.

(which corresponds to a volume $(V)$ of $33 \mathrm{fl}$ ) there will be $\sim 100$ molecules of RNase $\mathrm{H}, 4,000$ RNAPs and the lowest DNA copy numbers will be $\sim 1,600$.

Generating a population of oscillators in microdroplets. To study the effect of compartmentalization into small volumes on the dynamical behaviour of the transcriptional oscillator, we encapsulated the oscillator components within microemulsion droplets. Water-in-oil droplets were prepared using a non-ionic surfactant ${ }^{38}$, which resulted in microemulsions that were stable for days. A simple vortexing procedure generated a broad droplet-size distribution, with radii that ranged from $\sim 1 \mu \mathrm{m}$ to above $20 \mu \mathrm{m}$ (Methods). Owing to the nonionic nature of the surfactant, adsorption of molecules to the droplet boundaries was presumed to be very low. This was validated by control fluorescence measurements on emulsions prepared solely with 

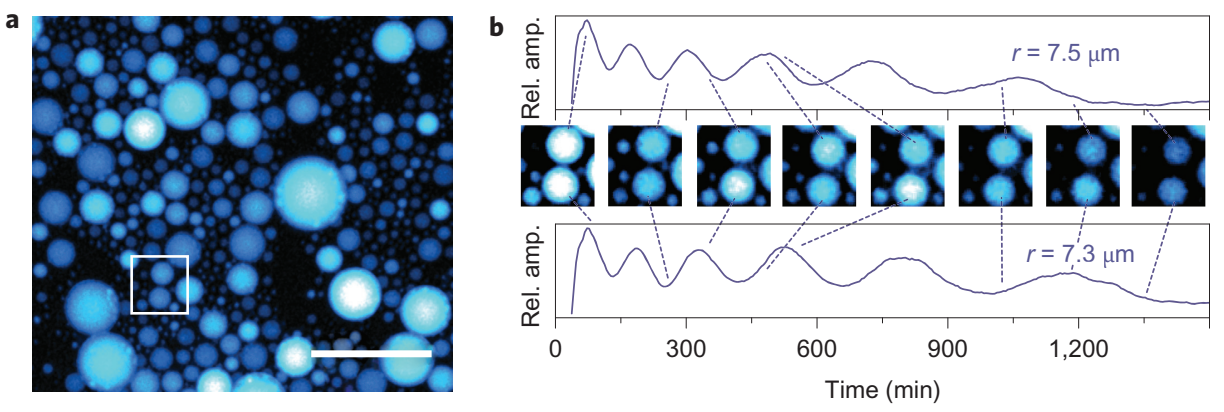

Figure 2 | Compartmentalized synthetic transcriptional oscillators. a, Epifluorescence microscopy image of the transcriptional oscillator in microemulsion droplets (scale bar, $100 \mu \mathrm{m}$ ); see also the example microscopy in Supplementary Videos V1-V3. b. Fluorescent time traces for two droplets that contain the same reaction mix as the bulk oscillator in Fig. 1. Sustained oscillations in droplets were observed for more than 20 hours. The droplet image series shows microscope snapshots in fluorescent mode during the oscillation reactions. The corresponding phases in the time traces are indicated by dashed lines. Rel. amp., relative amplitude.
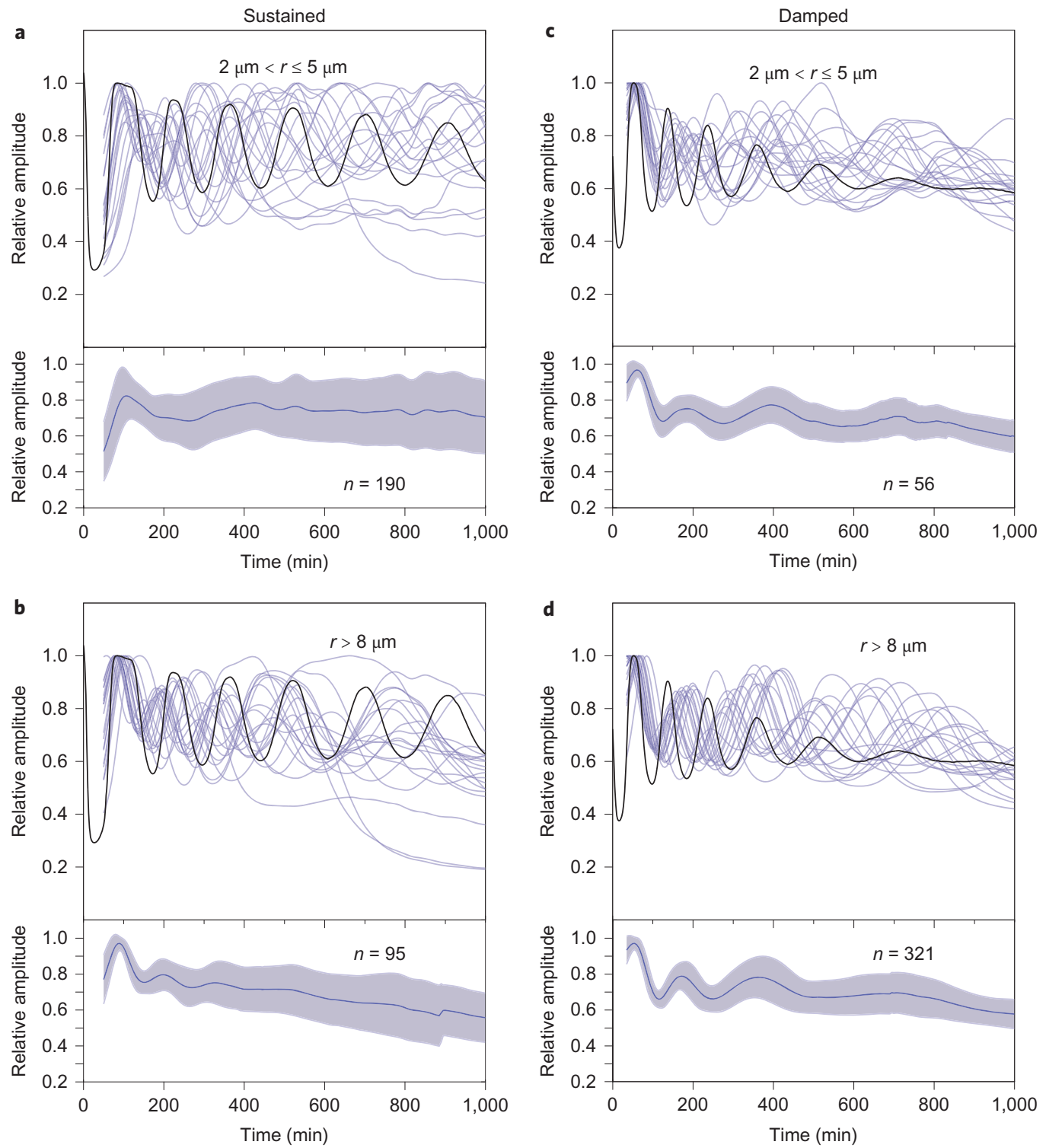

Figure 3 | Dynamical diversity in oscillatory behaviour caused by encapsulation of sustained and damped synthetic oscillator circuits. Only droplets that identifiably oscillate are considered here. Traces were normalized to their maximum value for this figure. a, Top panel, 20 example traces (blue) for the sustained oscillator in droplets with $r=2-5 \mu \mathrm{m}$ compared to the bulk oscillation trace (black). Bottom panel, population average of $n$ traces (blue) and corresponding standard deviation (indicated by the purple shaded area around the average). $\mathbf{b}$, As in $\mathbf{a}$, but for oscillators encapsulated in droplets with $r>8 \mu \mathrm{m}$. c, Top, 20 example traces (blue) for the damped oscillator $(r=2-5 \mu \mathrm{m})$ compared to the bulk oscillation trace (black). Bottom, population average of $n$ traces (blue) and corresponding standard deviation (purple shaded area). $\mathbf{d}$, As in $\mathbf{c}$, but for oscillators encapsulated in droplets with $r>8 \mu \mathrm{m}$. 

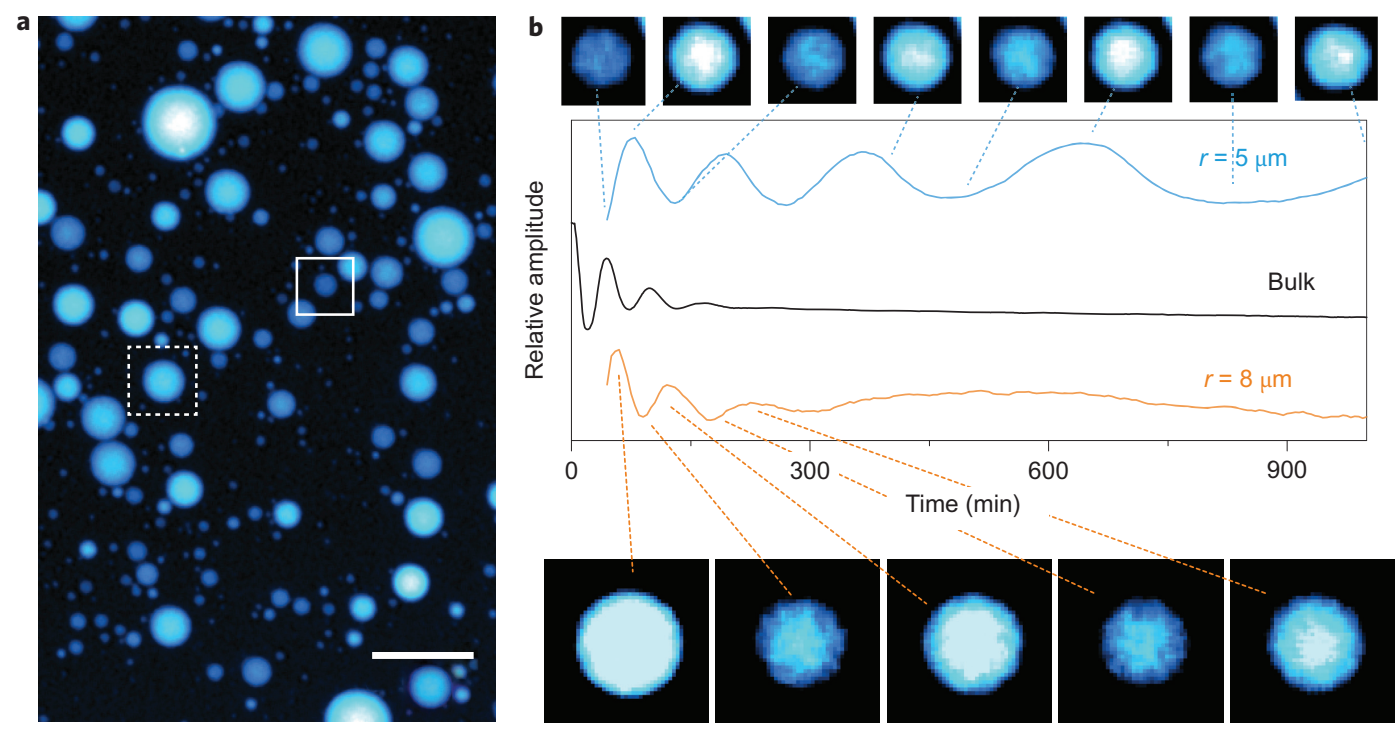

Figure 4 | Oscillations induced by compartmentalization. a, Population of droplets containing a reaction mixture that displays only strongly damped behaviour in bulk. The white solid box indicates the position of a droplet with $r \approx 5 \mu \mathrm{m}$ that displays sustained oscillations, and the dashed box indicates the position of a droplet that shows damped oscillations, with $r \approx 8 \mu \mathrm{m}$ (scale bar, $50 \mu \mathrm{m}$ ). $\mathbf{b}$, Fluorescence time trace recorded from the oscillating droplet from a (light blue), the strongly damped droplet (orange) and the strongly damped bulk signal (black). The bulk trace is classified as 'not identifiably oscillating' by our filtering algorithms. The $y$ axis represents the relative oscillation amplitude of the traces, which were scaled and shifted for clarity.

fluorescently labelled oligonucleotides and green fluorescent protein (Supplementary Section Compartmentalization).

Oscillations in microdroplets. For the initial experiments, we tuned the bulk oscillator into two dynamically distinct regimes by adjusting the RNase H concentration (see Supplementary Section Methods). In the first regime (identical to that shown in Fig. 1c), 'sustained' oscillations with minimal damping were observed, but in the other regime (with 25\% higher RNase $\mathrm{H}$ concentrations) the oscillator circuit produced 'damped' oscillations, which indicates that for this choice of parameters the bulk system was closer to the nonoscillatory region of its phase space. A fluorescence image of microdroplets that encapsulate oscillators in the sustained regime is displayed in Fig. 2a (see also Supplementary Videos V1-V3). As indicated by the example traces in Fig. $2 b$, oscillations differ in amplitude and frequency from droplet to droplet. To obtain a better quantitative picture of the diverse dynamical behaviour within the droplet populations, we tracked individual droplets and evaluated their dynamics as a function of size (Methods).

Figure 3 a shows 20 example oscillatory traces obtained in the sustained regime in 'small' droplets with radii in the range $r=2-5 \mu \mathrm{m}$ $(V=33-524 \mathrm{fl})$; the population average of the oscillatory signals together with the standard deviation around the mean value are also shown. Figure $3 \mathrm{~b}$ contains example and average traces for the sustained oscillator for 'large' droplets with radii $r>8 \mu \mathrm{m}(V>2 \mathrm{pl})$. Figure $3 \mathrm{c}$,d shows the corresponding plots for the damped oscillator (see Supplementary Section Oscillator Data and Supplementary Figs 5-11 for an overview of all data sets).

Of the traces that passed our filtering criteria (Supplementary Section Data Analysis and Supplementary Figs 2-4), only those with at least five clear extrema were defined as 'identifiably oscillating'. These may include both sustained oscillations and damped oscillations similar to the bulk traces in Fig. 3a,c; however, strongly damped traces and traces that show sustained but extremely slow oscillations may pass the filtering criteria, but would not be deemed 'identifiably oscillating'. Considering all identifiably oscillating traces (Fig. 3, lower panels), we observed that larger droplets have smaller variation for both oscillator tunings. In the sustained case, large droplets exhibit especially low variance at early times and the oscillating mean value indicates only slow desynchronization. Smaller droplets have greater initial variance and desynchronize more quickly. The 'dephasing' of the oscillations is less dramatic in the damped case (Fig. 3c,d), in which the standard deviation is considerably smaller than that for the sustained oscillator.

We reasoned that encapsulation might also 'induce' oscillations in a system that is barely oscillating in bulk. To test this hypothesis, we tuned the oscillator out of the oscillating region by an appropriate change in enzyme concentrations, which resulted in strongly damped behaviour in the bulk. Indeed, in this case sustained oscillations were observed in some of the smaller droplets (Fig. 4).

For traces identified as oscillating, in all three tunings we determined the oscillation periods as a function of droplet radius $r$, as shown in Fig. 5a-c. The oscillations tended to slow down with smaller droplet size as the variance of the periods increased. In the strongly damped case, identifiably oscillating droplets were observed only for $r \leq 10 \mu \mathrm{m}$; our filtering criteria and measurement noise precluded identification of oscillations with periods faster than $\sim 80$ minutes or of those with low amplitudes. An analysis of the corresponding amplitudes showed very similar tendencies, with typically increasing amplitudes and amplitude variability for smaller droplets (Fig. 5d-f). As potential origins of these behaviours, we considered the effect of stochastic reaction dynamics, the influence of partitioning on the molecule numbers within the droplets and a potential loss of enzyme activity during compartmentalization.

Stochastic reaction dynamics. Stochastic reaction dynamics is expected to play a role for very small reaction volumes and molecule numbers. For instance, the oscillator species with the lowest concentration, RNase $\mathrm{H}$, would be present at a copy number of only $\sim 5$ for $V=1 \mathrm{fl}$. Indeed, stochastic simulations of the dynamics of the transcriptional oscillator using Gillespie's algorithm ${ }^{6}$ showed strong fluctuations for $V=1 \mathrm{fl}$ (Supplementary Fig. 29). However, by $r=2 \mu \mathrm{m}(V=33 \mathrm{fl})$ these fluctuations had already reduced strongly (Fig. 6a). For larger droplets the fluctuations were negligible, which indicates that stochastic reaction dynamics is not sufficient to explain the diversity observed in our droplet populations (Fig. 6b). 

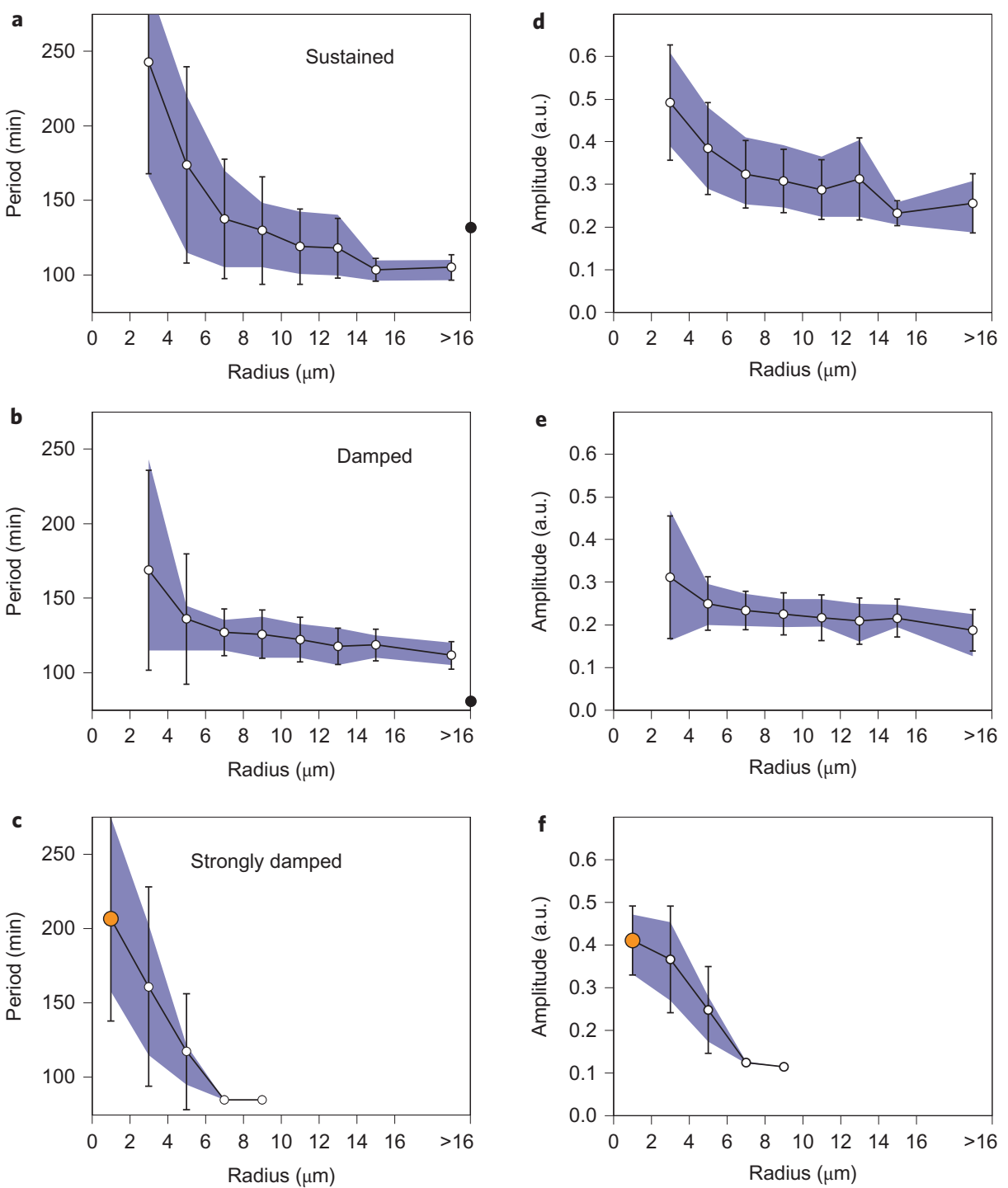

Figure 5 | Variation of oscillation periods and amplitudes with droplet radius for the sustained, damped and strongly damped oscillator tunings. a-c, Dependence of mean oscillation period on droplet radius. Bars indicate the standard deviation, whereas the shaded areas represent the range in which $60 \%$ of the population resides. Black dots on the right-hand side of $\mathbf{a}$ and $\mathbf{b}$ indicate the behaviour (period) of the bulk reactions. $\mathbf{d}-\mathbf{f}$, Corresponding amplitudes as functions of radius. The analysis is based on a total number of 1,193, 978 and 644 identifiably oscillating droplets for the sustained, damped and strongly damped tunings, respectively. The smallest data bins in $\mathbf{c}$ and $\mathbf{f}$ (orange) only contain a few droplets slightly below $r=2 \mu \mathrm{m}$. a.u., arbitrary units.

Partitioning effects. A major source of variability in our droplet populations may be partitioning effects. Small droplets of size $V$ contain molecule numbers $N$ that are expected to be Poisson distributed according to $p(N)=\left(\lambda^{N} / N !\right) \mathrm{e}^{-\lambda}$ where the parameter $\lambda=c V N_{\mathrm{A}}$ is the expected number of molecules for a bulk molar concentration $c$ and $N_{\mathrm{A}}$ is Avogadro's constant. Poisson partitioning also predicts that the standard deviation of $N$ will be $N^{1 / 2}$. Although the number fluctuations and corresponding concentration fluctuations are presumably small for most of the oscillator species (for example, $<10 \%$ for $\lambda>100$ ), the combination of the variations of all 24 species concentrations, amplified by the oscillator circuit, may well lead to considerable variability in the dynamics of the system.

To assess the influence of such partitioning effects, we performed simulations for varying droplet sizes using deterministic mass-action differential equations, for which the initial molecule numbers of all oscillator species were drawn from a Poisson distribution. The results of such simulations for a droplet radius of $r=2 \mu \mathrm{m}$ are shown in Fig. 6a. The simulations indicate that partitioning can, indeed, result in a qualitatively similar variability in dynamical behaviour to that observed experimentally. As shown in Fig. 6c and Supplementary Section Modelling, the mean period of the oscillations, as well as the variance, increases with decreasing droplet radius. Quantitatively, however, the effect of Poisson partitioning is weaker than that in the experiment, in which strong variability is observed even for relatively large droplets.

Broader-than-Poisson variability. This strong variability could be explained by several factors, such as a small fraction of enzymes being active, incomplete mixing of the solution, protein multimerization or aggregation that results in copy-number variance greater than the mean ${ }^{9}$ or stochastic protein inactivation because of adsorption on the droplet interface and denaturation during generation of the microdroplets ${ }^{39}$. In principle, determination of concentrations within individual droplets could assess directly whether partitioning was Poisson or broader, but our fluorescence measurements did not provide concentration values with sufficient accuracy (Supplementary Section Compartmentalization and 
a

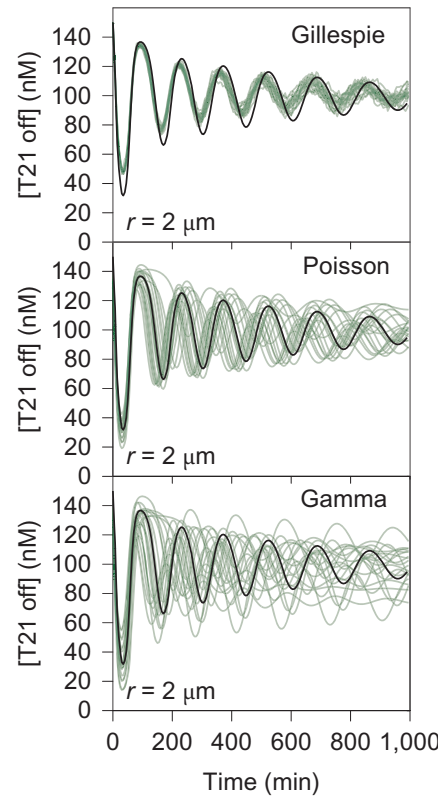

b

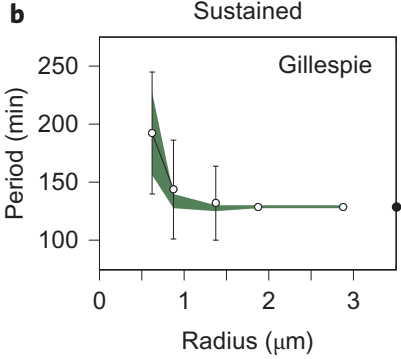

c

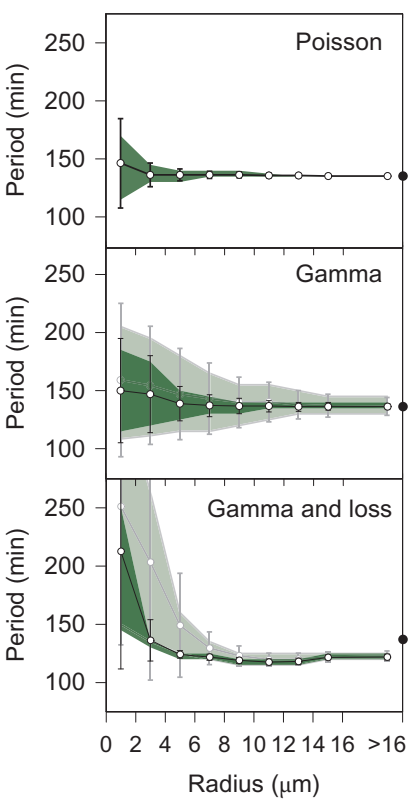

d

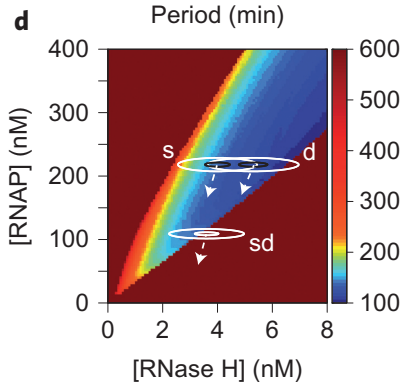

Damped
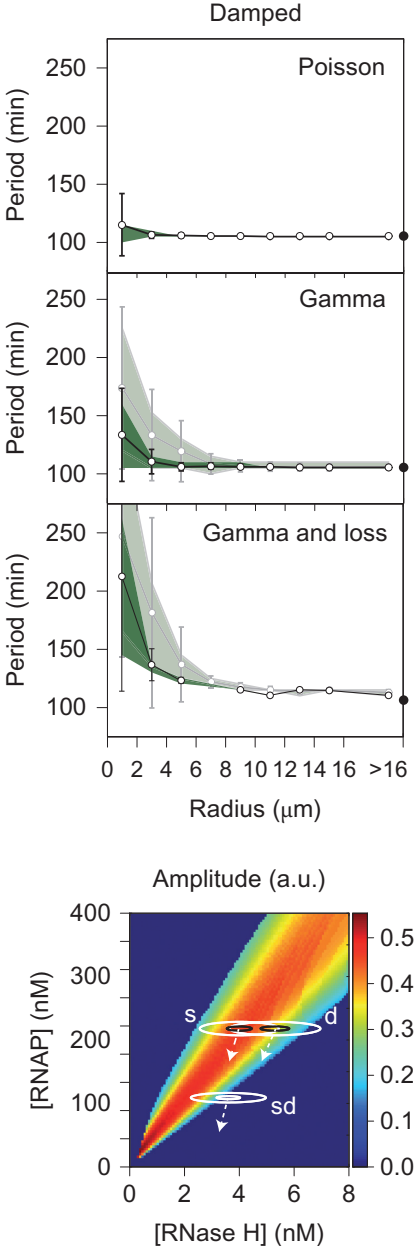

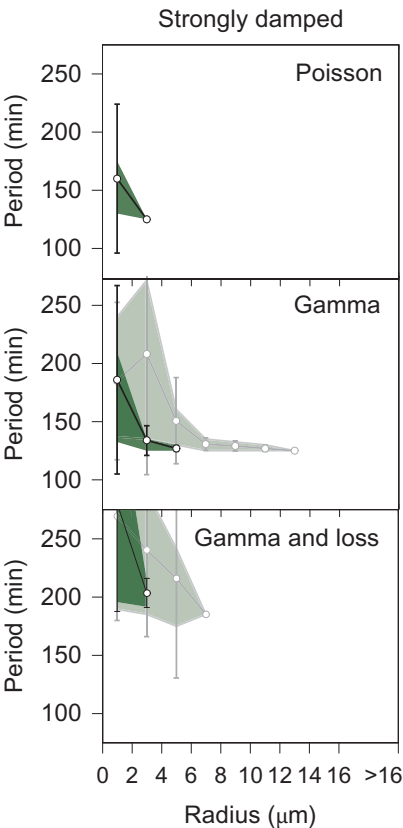

Damping coefficient $\left(\mathrm{h}^{-1}\right)$

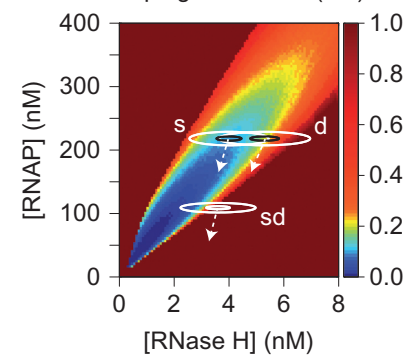

Figure 6 | Numerical modelling of the compartmentalized oscillator. a, Concentrations of oscillator species T21 for $r=2 \mu m$ droplet radii simulated stochastically using Gillespie's algorithm (without 'partitioning noise') (top), and deterministically with varying initial conditions chosen according to Poisson statistics (middle) and gamma statistics (with $\beta=10$ ) (bottom), all based on the sustained oscillation tuning. $\mathbf{b}$, Distribution of periods as a function of droplet radius for stochastic simulations without partitioning noise. $\mathbf{c}$, Distribution of oscillation periods as a function of radius for Poisson and gamma ( $\beta=10$, dark green; $\beta=100$, light green) partitioning noise, as well as additional loss of enzyme activity during droplet production, simulated for the sustained tuning (left), the damped tuning (middle) and the strongly damped tuning (right). The bulk oscillation period is marked as a dot on the right axis for the sustained and damped tunings; for the strongly damped tuning, the bulk reaction did not identifiably oscillate. For RNase $\mathrm{H}$ a global loss of $~ 10 \%$ was assumed, whereas RNAP activity varied from $63 \%$ of the bulk value at $r=10 \mu \mathrm{m}$ to $42 \%$ at $r=2 \mu \mathrm{m}$ (see Supplementary Section Modelling). Error bars in b and $\mathbf{c}$ denote standard deviations. Shaded areas are between the $20 \%$ and $80 \%$ quantiles. d, Phase diagrams calculated from the oscillator model that display period, amplitude and damping coefficient of the oscillations as a function of RNAP and RNase $\mathrm{H}$ concentrations. Also indicated are the (bulk) initial conditions for the sustained (s), damped (d) and strongly damped (sd) cases. Small black or white ellipses indicate the standard deviation expected from Poisson statistics, and large white ellipses show the standard deviation for the gamma distribution $(\beta=10)$ for droplets with $r=2 \mu \mathrm{m}$ radius. Dashed arrows indicate the effect of enzyme loss.

Supplementary Figs 13 and 14). Instead, we examined the variability in two 'single-enzyme subsystems' (Supplementary Fig. 15): one consisted of just RNase $\mathrm{H}$ acting on an RNA/DNA substrate, in which an enzyme turnover of $\sim 100$ effectively amplified the signal that resulted from partitioning; the other contained only RNAP, a transcription template, and a sensor for the fluorescent readout. In both cases, the apparent variability in enzyme activity was an order of magnitude larger than would be expected from Poisson partitioning combined with measurement noise (Supplementary Section Compartmentalization). Furthermore, independent of the droplet radius, the median of RNase $\mathrm{H}$ activity seemed to be only slightly reduced compared to that of the bulk case (Supplementary Fig. 17). By contrast, RNAP activity was considerably lower for droplets with smaller radii (Supplementary Fig. 16), which can be explained qualitatively by a loss of enzyme function during the droplet-generation process (see Supplementary Sections
Compartmentalization and Modelling). This may be caused by enzyme aggregation, which we also observed for fluorescently labelled T7 RNAP encapsulated into the emulsion droplets (Supplementary Fig. 12) ${ }^{40}$.

We modelled empirically the larger variability with a gamma distribution, whose probability density function for $x \geq 0$ is given by

$$
p(x ; \alpha, \beta)=\left(\beta^{\alpha} \Gamma(\alpha)\right)^{-1} x^{\alpha-1} e^{-x / \beta}
$$

where $\Gamma(\alpha)$ is the gamma function, $\alpha$ is the 'shape factor' and $\beta$ is the 'scale factor'. This distribution allowed us to choose independently the mean $\langle x\rangle=\alpha \beta$ and variance $\operatorname{var}(x)=\langle x\rangle \beta$ of the distribution. Previously, the gamma distribution was used in the context of stochastic gene expression, when gene expression 'bursts' were considered together with the influence of cell division and degradation processes ${ }^{41}$. However, a variety of different scenarios for 
cellular heterogeneity led to essentially the same 'gamma-like' molecular-number distributions ${ }^{35}$.

In the revised simulations, the initial molecule numbers for each species in a droplet were drawn from a gamma distribution with mean $\langle x\rangle=c V N_{\mathrm{A}}$ and scale factors $\beta=10$ or $\beta=100$, which span the range of $\beta$ values suggested by the single-enzyme experiments (Supplementary Figs 16 and 19). As shown in Fig. $6 a, c$, the simulations reproduce the dynamical diversity of the experiments substantially better (Supplementary Section Modelling and Supplementary Figs 23-28). Particularly, the model captures qualitatively the change of size-dependent variability for different oscillator tuning: the sustained tuning has a wider range of radii that exhibit significant variability compared with the damped tuning, the strongly damped tuning yields oscillations only for smaller droplets and, in all three tunings, the mean period increases slightly for very small droplets. As also shown in Fig. 6c, simulations that take the observed enzyme loss into account capture this trend towards larger periods for small droplet radii even better.

If we assume that variations in active-enzyme concentrations are the dominant sources of variability in our experiments, we can understand semiquantitatively the observed trends summarized in Fig. 5 on the basis of two-dimensional 'phase diagrams'. In Fig. 6d, the oscillator period, amplitude and damping coefficients predicted from our numerical model are shown as a function of [RNase $\mathrm{H}]$ and [RNAP]. As indicated in the plots, depending on the position of the initial conditions in phase space and also on the width of their distribution, a wide range of oscillations with different periods or amplitudes are sampled, even the non-oscillatory dynamics. This also explains the different behaviour observed for the sustained, damped and strongly damped oscillator tunings. Whereas variations towards smaller enzyme concentrations can drive the sustained oscillator into a phase-space region with large periods, this effect is expected to be less pronounced for the damped oscillator, whose (bulk) initial conditions happen to be in a less-sensitive region of phase space. Furthermore, Fig. 6d also shows that fluctuations can drive the strongly damped oscillator into the oscillatory region. In addition, an overall loss of enzymes shifts the bulk initial conditions towards lower values.

\section{Conclusion}

In our study of a compartmentalized in vitro transcriptional oscillator we found significant deviations from the bulk system and a broad diversity in dynamical behaviour. The dynamics of the encapsulated system can be understood as the result of a broad distribution in initial concentrations amplified by the underlying dynamical system, and can be reproduced adequately in deterministic simulations with statistically varying initial conditions. According to our model, we found that for the relatively large molecule numbers studied here, the inherent stochasticity of chemical reactions cannot account for the variability. Adopting the language of stochastic gene expression, our system appears to be dominated by 'extrinsic' sources of noise, such as enzyme-number variability and partitioning effects ${ }^{35,36}$, rather than 'intrinsic' noise caused by stochastic reaction dynamics ${ }^{33,34}$.

Our experiments and numerical analyses suggest that the physical sources of variability include stochastic partitioning of molecules and loss of enzyme activity during the droplet-production process. These phenomena yield broader distributions of molecule numbers, and thus dynamic variability among droplet oscillations, which is captured qualitatively by our gamma partitioning model. How the variability in initial conditions is propagated through the dynamics is affected profoundly by the operating point of the oscillator.

For biological cells, it has been argued previously that the influence of partitioning noise may be underappreciated compared to that of stochastic reaction dynamics ${ }^{35,36}$. Our study suggests that similar 'partitioning effects' play a major role in determining the dynamic behaviour of compartmentalized artificial biochemical circuits: these effects pose a major challenge for cell-scale molecular engineering and the realization of protocells. Therefore, it is important to investigate which synthetic circuits provide better or worse performance in the presence of such noise, and to study and potentially adopt strategies of biological cells for either active noise reduction or increased resilience ${ }^{27-29}$.

An interesting application of compartmentalization-induced variability could be the deliberate generation of widely varying initial concentrations for the exploration and characterization of the dynamical potential of other nonlinear biochemical networks. If the initial concentrations of key species could be measured directly in each droplet, then the resulting behaviour of dynamical species could be associated directly with the initial concentrations, thus mapping the phase diagram in a single experiment. Taking our oscillator as an example, if RNAP and RNase $\mathrm{H}$ could be labelled individually ${ }^{42}$, and if measurement accuracy could be improved, then microscopy of droplet microemulsions could provide a highthroughput experimental measurement of phase diagrams, analogous to those of Fig. $6 \mathrm{~d}$, as a function of droplet radius.

As our programmable biochemical systems can be used to design complex chemical networks with a well-defined topology, an exciting opportunity for quantitative analysis of such networks would be to use the whole range of observed dynamical behaviours for parameter estimation, for example by fitting each trace obtained from an experiment with the same set of rate parameters, but with differing initial conditions. This collective data-fitting approach is argued to yield models with more predictive power than if each parameter is measured individually with similar accuracy ${ }^{43,44}$, but it is limited by the availability of extensive data sets. Such high-throughput methods for characterizing synthetic circuit behaviours (potentially supported by microfluidic liquid handling techniques ${ }^{10}$ ) will probably prove essential for the development of sophisticated but stochastic cell-scale artificial molecular systems.

\section{Methods}

DNA oligonucleotides and enzymes. Oligonucleotides were purchased from IDT DNA or biomers.net. RNase $\mathrm{H}$ was purchased from Applied Biosystems. T7 RNAP and reagents from Epicentre and New England Biolabs were used for the preparation of the transcription mix. DNA sequences, modifications and details on sample preparation and experimental conditions are given in the Supplementary Methods.

Fluorescence measurements. Bulk fluorescence experiments were performed on a Horiba/Jobin Yvon Fluorolog 3 system in $45 \mu \mathrm{l}$ cuvettes. Fluorescence emission from labelled DNA strands was recorded every minute. A sample temperature of $37^{\circ} \mathrm{C}$ was maintained using a water-circulation thermostat.

Generation of microemulsion droplets. Two alternative methods for the generation of the microdroplets were employed. The first method, which we termed 'shakennot-stirred', consisted of simply vortexing a mixture of oil, surfactant and aqueous buffer solution that contained the reagents (Supplementary Methods). By adjusting mixing time and vibration speed appropriately, we could generate populations of droplets with a broad size distribution, with radii that ranged from $\sim 1 \mu \mathrm{m}$ to $>20 \mu \mathrm{m}$ (see Supplementary Section Oscillator Data). Droplets were generated by mixing $10 \mu \mathrm{l}$ oscillator reaction mix with $45 \mu \mathrm{FC}-40$ Fluorinert oil (F9755, SigmaAldrich) that contained $1.8 \%$ (w/w) E2K0660 non-ionic, biocompatible surfactant (RainDance Technologies) in Protein LoBind tubes (Eppendorf) using a vortex mixer for 60 seconds. A volume of $45 \mu \mathrm{l}$ emulsion was transferred with a pipette from the reaction tube into ibidi $\mu$-slides $\mathrm{VI}^{0.4}$ for microscope measurements. The flow chambers were sealed with PCR tape to protect the sample against evaporation. We also employed microfluidics that generate emulsions: this allowed us to produce large numbers of droplets with a much narrower size distribution (see Supplementary Methods), but it was technically challenging to produce very small droplets with diameters as small as $2 \mu \mathrm{m}$. The shaken-not-stirred method, similar to common emulsion PCR techniques ${ }^{45}$, allowed us to obtain, in a single experiment, populations of droplets with broad size variability, and a large number of droplets with radii in the range $2-3 \mu \mathrm{m}$. For this reason, we adopted the shaken-not-stirred method for all experiments reported in this paper, except for those reported in the Supplementary Methods (Supplementary Fig. 1). 
Time-lapse microscopy. This was conducted on an Olympus IX81 epifluorescence microscope equipped with an automated $x-y$ stage (Prior Scientific) and an incubator box $\left(T=37^{\circ} \mathrm{C}\right)$, and controlled with MicroManager 1.4. Every five minutes, a bright field and a fluorescence image were recorded for at least 1,000 minutes.

Image analysis and data processing. Droplets were tracked automatically and analysed from each microscope movie (see Supplementary Section Data Analysis and Supplementary Videos V1-V3). Fluorescence values were normalized with respect to a reference dye present at a constant concentration in the droplets, and smoothed to reduce noise. To filter out artefacts, stringent selection criteria were used to remove anomalous traces deemed likely to result from, for example, failures in droplet tracking or the superposition of droplets. These criteria also remove some genuine droplet traces that exhibit low-amplitude oscillations or non-oscillatory behaviour; consequently, we do not report the fraction of droplets that oscillate, as this measurement is unreliable. Additional details are given in Supplementary Section Data Analysis.

Received 24 October 2012; accepted 13 January 2014; published online 16 February 2014; corrected after print 22 April 2014

\section{References}

1. Kim, J., White, K. S. \& Winfree, E. Construction of an in vitro bistable circuit from synthetic transcriptional switches. Mol. Sys. Biol. 2, 68 (2006).

2. Forster, A. C. \& Church, G. M. Synthetic biology projects in vitro. Genome Res. 17, 1-6 (2007).

3. Montagne, K., Plasson, R., Sakai, Y., Fujii, T. \& Rondelez, Y. Programming an in vitro DNA oscillator using a molecular networking strategy. Mol. Syst. Biol. 7, 466 (2011).

4. Zhou, H-X., Rivas, G. \& Minton, A. P. Macromolecular crowding and confinement: biochemical, biophysical, and potential physiological consequences. Ann. Rev. Biophys. 37, 375-397 (2008).

5. De, T. \& Maitra, A. Solution behaviour of aerosol OT in non-polar solvents. Adv. Colloid Interface Sci. 59, 95-193 (1995).

6. Gillespie, D. T. Exact stochastic simulation of coupled chemical reactions. J. Phys. Chem. 81, 2340-2361 (1977).

7. Noireaux, V. \& Libchaber, A. A vesicle bioreactor as a step toward an artificial cell assembly. Proc. Natl Acad. Sci. USA 101, 17669-17674 (2004).

8. Shin, J. \& Noireaux, V. An E. coli cell-free expression toolbox: application to synthetic gene circuits and artificial cells. ACS Synthetic Biol. 1, 29-41 (2012).

9. Matsuura, T. et al. Effects of compartment size on the kinetics of intracompartmental multimeric protein synthesis. ACS Synthetic Biol. 1, 431-437 (2012).

10. Theberge, A. B. et al. Microdroplets in microfluidics: an evolving platform for discoveries in chemistry and biology. Angew. Chem. Int. Ed. 49, 5846-5868 (2010).

11. Rotman, B. Measurement of activity of single molecules of beta-D-galactosidase Proc. Natl Acad. Sci. USA 47, 1981-1991 (1961).

12. Nakano, M. et al. Single-molecule PCR using water-in-oil emulsion. J. Biotechnol. 102, 117-124 (2003).

13. Miller, O. J. et al. Directed evolution by in vitro compartmentalization. Nature Methods 3, 561-570 (2006).

14. Lu, W-C. \& Ellington, A. D. In vitro selection of proteins via emulsion compartments. Methods 60, 75-80 (2013).

15. Epstein, I. et al. Chemical oscillators in structured media. Acc. Chem. Res. 45, 2160-2168 (2011).

16. Nagypal, I. \& Epstein, I. R. Fluctuations and stirring rate effects in the chlorite thiosulfate reaction. J. Phys. Chem. 90, 6285-6292 (1986).

17. Vanag, V. \& Epstein, I. Pattern formation in a tunable medium: the BelousovZhabotinsky reaction in an aerosol OT microemulsion. Phys. Rev. Lett. 87, 228301 (2001).

18. Elowitz, M. B. \& Leibler, S. A synthetic oscillatory network of transcriptional regulators. Nature 403, 335-338 (2000).

19. Fung, E. et al. A synthetic gene-metabolic oscillator. Nature 435, 118-122 (2005).

20. Stricker, J. et al. A fast, robust and tunable synthetic gene oscillator. Nature 456, 516-539 (2008).

21. Tigges, M., Marquez-Lago, T. T., Stelling, J. \& Fussenegger, M. A tunable synthetic mammalian oscillator. Nature 457, 309-312 (2009).

22. Danino, T., Mondragón-Palomino, O., Tsimring, L. \& Hasty, J. A synchronized quorum of genetic clocks. Nature 463, 326-330 (2010)

23. Ackermann, J., Wlotzka, B. \& McCaskill, J. In vitro DNA-based predator-prey system with oscillatory kinetics. Bull. Math. Biol. 60, 329-354 (1998).
24. Kim, J. \& Winfree, E. Synthetic in vitro transcriptional oscillators. Mol. Syst. Biol. 7, 465 (2011).

25. Franco, E. et al. Timing molecular motion and production with a synthetic transcriptional clock. Proc. Natl Acad. Sci. USA 108, E784-E793 (2011).

26. Winfree, A. T. The Geometry of Biological Time (Springer, 1980).

27. Gonze, D., Halloy, J. \& Goldbeter, A. Robustness of circadian rhythms with respect to molecular noise. Proc. Natl Acad. Sci. USA 99, 673-678 (2002).

28. Vilar, J. M. G., Kueh, H. Y., Barkai, N. \& Leibler, S. Mechanisms of noiseresistance in genetic oscillators. Proc. Natl Acad. Sci. USA 99, 5988-5992 (2002).

29. Kar, S., Baumann, W. T., Paul, M. R. \& Tyson, J. J. Exploring the roles of noise in the eukaryotic cell cycle. Proc. Natl Acad. Sci. USA 106, 6471-6476 (2009).

30. Hong, C. I., Conrad, E. D. \& Tyson, J. J. A proposal for robust temperature compensation of circadian rhythms. Proc. Natl Acad. Sci. USA. 104, 1195-1200 (2007)

31. Fujii, T. \& Rondelez, Y. Predator-prey molecular ecosystems. ACS Nano 7, 27-34 (2013).

32. Hasatani, K. et al. High-throughput and long-term observation of compartmentalized biochemical oscillators. Chem. Comm. 49, 8090-8092 (2013).

33. Kaern, M., Elston, T. C., Blake, W. J. \& Collins, J. J. Stochasticity in gene expression: from theories to phenotypes. Nature Rev. Genet. 6, 451-464 (2005).

34. Raj, A. \& van Oudenaarden, A. Nature, nurture, or chance: stochastic gene expression and its consequences. Cell 135, 216-226 (2008).

35. Huh, D. \& Paulsson, J. Non-genetic heterogeneity from stochastic partitioning at cell division. Nature Genet. 43, 95-100 (2011).

36. Huh, D. \& Paulsson, J. Random partitioning of molecules at cell division. Proc. Natl Acad. Sci. USA 108, 15004-15009 (2011).

37. Rizzo, J., Gifford, L. K., Zhang, X., Gewirtz, A. M. \& Lu, P. Chimeric RNA-DNA molecular beacon assay for ribonuclease $H$ activity. 16, 277-283 (2002).

38. Holtze, C. et al. Biocompatible surfactants for water-in-fluorocarbon emulsions. Lab on a Chip 8, 1632-1639 (2008).

39. Liu, Y., Jung, S-Y. \& Collier, C. P. Shear-driven redistribution of surfactant affects enzyme activity in well-mixed femtoliter droplets. Anal. Chem. 81, 4922-4928 (2009).

40. Maslak, M. \& Martin, C. T. Effects of solution conditions on the steady-state kinetics of initiation of transcription by T7 RNA polymerase. Biochemistry 33, 6918-6924 (1994)

41. Friedman, N., Cai, L. \& Xie, X. S. Linking stochastic dynamics to population distribution: an analytical framework of gene expression. Phys. Rev. Lett. 97 (2006).

42. Modesti, M. in Single Molecule Analysis (eds Peterman, E. J. G. \& Wuite, G. J. L.) 101-120 (Methods in Molecular Biology 783, Humana Press, 2011).

43. Gutenkunst, R. N. et al. Universally sloppy parameter sensitivities in systems biology models. PLoS Comput. Biol. 3, e189 (2007).

44. Subsoontorn, P., Kim, J. \& Winfree, E. Ensemble Bayesian analysis of bistability in a synthetic transcriptional switch. ACS Synthetic Biol. 1, 299-316 (2012).

45. Schütze, T. et al. A streamlined protocol for emulsion polymerase chain reaction and subsequent purification. Anal. Biochem. 410, 155-157 (2011).

\section{Acknowledgements}

The authors acknowledge financial support by the National Science Foundation grants CCF-0832824 (The Molecular Programming Project) and CMMI-1266402, by the Bourns College of Engineering at the University of California at Riverside (UC), the UC Regents Faculty Development Fellowship, the European Commission FP7 grant no. 248919 (Bacterial Computing with Engineered Populations), the German Research Foundation Cluster of Excellence Nanosystems Initiative Munich and the Elite Network of Bayern. Surfactant E2K0660 was supplied by RainDance Technologies. We acknowledge E. Friedrichs and R. Jungmann for initial experiments; U. Gerland, R. Murray and N. Karlsson for useful discussions, advice and support; and C. Martin, L. Ramirez-Tapia, E. Stahl and H. Dietz for providing fluorescently labelled T7 RNAP.

\section{Author contributions}

E.W., E.F. and F.C.S. designed the research; M.W., J.K. and E.F. performed the research; M.W., J.K., K.K. and E.F. analysed the data; E.W., E.F. and F.C.S wrote the paper.

\section{Additional information}

Supplementary information is available in the online version of the paper. Reprints and permissions information is available online at www.nature.com/reprints. Correspondence and requests for materials should be addressed to E.F. and F.C.S.

\section{Competing financial interests}

The authors declare no competing financial interests. 


\section{ADDENDUM}

\section{Diversity in the dynamical behaviour of a compartmentalized programmable biochemical oscillator}

Maximilian Weitz, Jongmin Kim, Korbinian Kapsner, Erik Winfree, Elisa Franco and Friedrich C. Simmel

Nature Chemistry 6, 295-302 (2014); Published online 16 February 2014; corrected after print 22 April 2014.

The authors wish to add the following to the Acknowledgements section of this Article 'E.F. and F.C.S. gratefully acknowledge funding from the Bavaria California Technology Center (BaCaTeC).' The online versions of the Article have been amended accordingly. 